\title{
The new CGEM Inner Tracker and the new TIGER ASIC for the BES III Experiment
}

\author{
S. Marcello, ${ }^{*}$ tab M. Alexeev, ${ }^{a, b}$ A. Amoroso, ${ }^{a, b}$ R. Baldini Ferroli, ${ }^{c, d}$ M. Bertani, ${ }^{c}$ \\ D. Bettoni, ${ }^{e}$ F. Bianchi, ${ }^{\text {a,b }}$ A. Calcaterra, ${ }^{\mathrm{c}}$ N. Canale, ${ }^{\mathrm{e}}$ M. Capodiferro, ${ }^{\mathrm{c}, \mathrm{f}}$ V. Carassiti, ${ }^{\mathrm{e}}$ \\ S. Cerioni, ${ }^{\mathrm{c}}$ JY. Chai, ${ }^{\mathrm{b}, \mathrm{d}, \mathrm{g}}$ S. Chiozzi, ${ }^{\mathrm{e}}$ G. Cibinetto, ${ }^{\mathrm{e}}$ F. Cossio, ${ }^{\mathrm{b}, \mathrm{g}}$ A. Cotta \\ Ramusino, ${ }^{e}$ G. Cotto, ${ }^{a b}$ M. Da Rocha Rolo, ${ }^{b}$ F. De Mori, ${ }^{a, b} M$. Destefanis, ${ }^{a, b}$ \\ J. Dong, ${ }^{\mathrm{c}}$ F. Evangelisti, ${ }^{\mathrm{e}}$ R. Farinelli, ${ }^{\mathrm{e}}, \mathrm{h}$ L. Fava, ${ }^{\mathrm{b}}$ G. Felici, ${ }^{\mathrm{c}} \mathrm{I}$. Garzia, ${ }^{\mathrm{e}, \mathrm{h}} \mathrm{M}$. Gatta, ${ }^{\mathrm{c}}$ \\ M. Greco, ${ }^{a, b}$ L. Lavezzi, ${ }^{b, d}$ CY. Leng, ${ }^{b, d, g}$ H. Li, ${ }^{b, d}$ M. Maggiora, ${ }^{a, b}$ R. Malaguti, ${ }^{e}$ \\ M. Melchiorri, ${ }^{\mathrm{e}}$ G. Mezzadri, ${ }^{\mathrm{e}, \mathrm{h}}$ M. Mignone, ${ }^{\mathrm{b}}$ G. Morello, ${ }^{\mathrm{c}}$ S. Pacetti, ${ }^{\mathrm{i}, \mathrm{l}}$ P. Patteri, ${ }^{\mathrm{c}}$ \\ J. Pellegrino, ${ }^{a, b}$ A. Pelosi, ${ }^{c}$ A. Rivetti, ${ }^{b}$ M. Savrié,, ${ }^{e, h}$ E. Soldani, ${ }^{c}$ S. Sosio, ${ }^{a, b}$ \\ S. Spataro, ${ }^{a, b}$ E. Tskhadadze, ${ }^{c, m}$ C. Weishuai, ${ }^{b, d, g}$ R. Wheadon, ${ }^{b}$ L. Yan ${ }^{b}$
}

${ }^{a}$ Dipartimento di Fisica, Università di Torino, Via P. Giuria 1, Torino, 10125, Italy

${ }^{b}$ INFN Sezione di Torino, Via P. Giuria 1, Torino, 10125, Italy

'INFN Laboratori Nazionali di Frascati, Via E. Fermi 40, Frascati (Roma), 00044, Italy

${ }^{d}$ Institute of High Energy Physics, Chinese Academy of Sciences, 19B Yuquan Lu, Beijing, 100049, P.R.C.

eINFN Sezione di Ferrara, Via G. Saragat 1, Ferrara, 44122, Italy

fINFN Sezione di Roma, P.le A. Moro 2, Roma, 00185, Italy

${ }^{g}$ Dipartimento di Elettronica e Telecomunicazioni, Politecnico di Torino, C.so Duca degli Abbruzzi 24, Torino, 10129, Italy

${ }^{h}$ Dipartimento di Fisica, Università di Ferrara, Via G. Saragat 1, Ferrara, 44122, Italy

iINFN Sezione di Perugia, Via A. Pascoli 14, Perugia, 06123, Italy

'Dipartimento di Fisica e Geologia, Università di Perugia, Via A. Pascoli 14, Perugia, 06123, Italy

${ }^{m}$ Joint Institute for Nuclear Research (JINR), Joliot-Curie 6, Dubna, Moscow Region, 14980, Russia

\begin{abstract}
A new detector exploiting the technology of Gas Electron Multipliers is under construction to replace the innermost drift chamber of BESIII experiment, since its efficiency is compromised owing the high luminosity of Beijing Electron Positron Collider. The new inner tracker with a cylindrical shape will deploy several new features. The analogue readout and two complementary algorithms to reconstruct the position will allow achieving a spatial resolution of $130 \mu \mathrm{m}$ in a $1 \mathrm{~T}$ magnetic field. For this purpose, TIGER, a new custom 64-channel ASIC, providing time and charge measurements, has been developed. Here, a summary of the most recent results on detector and electronics prototypes is given.
\end{abstract}

EPS-HEP2017, European Physical Society conference on High Energy Physics

5-12 July 2017

Venice, Italy

\footnotetext{
*Speaker

†E-mail: simonetta.marcello@unito.it

(c) Copyright owned by the author(s) under the terms of the Creative Commons 


\section{Introduction}

The BESIII experiment [1] has been operating since 2009 at the BEPCII double-ring $\mathrm{e}^{+} \mathrm{e}^{-}$collider in Beijing, collecting a very large data sample in the center-of-mass energy region between 2 and $4.6 \mathrm{GeV}$, which allows an investigation of different topics such as charmonium, charm physics, hadron physics [2], and $\tau$-physics. In the last years, owing the high luminosity of $1.0 \times 10^{33} \mathrm{~cm}^{-2} \mathrm{~s}^{-1}$ achieved at BEPCII, the efficiency of the innermost drift chamber began to deteriorate [3]. The plan for the future is to continue data taking at least until the 2022. For this reason, a new inner tracker with a cylindrical shape, exploiting the technology of Gas Electron Multipliers (GEM), has been proposed to replace the current one. Specific requirements have been fixed for the new inner tracker; in particular, the $r \phi$ spatial resolution should be within $130 \mu \mathrm{m}$, operating in a high magnetic field of $1 \mathrm{~T}$.

\section{The Cylindrical GEM detector}

The cylindrical GEM inner tracker (CGEM-IT) for BESIII experiment consists of three concentric layers of triple GEMs, a detailed description can be found in Ref. 4 and 5.

The first cylindrical GEM detector with four layers has been constructed for the KLOE-2 experiment [6] and operated in a magnetic field of $0.5 \mathrm{~T}$, achieving a spatial resolution of $350 \mu \mathrm{m}$. The CGEM-IT project implements three new important features: the use of Rohacell 31 foam for the mechanical structure, which reduces the material budget of the three layers to a radiation length $\sim 1 \%$ of $\mathrm{X}_{0}$; a jagged strip layout of the anode which reduces the capacitance couplings between strips by $\sim 30 \%$; and the analogue readout of the strips, which allows loosening of the pitch size to $650 \mu \mathrm{m}$, using a limited number of channels ( 10 000 instead of 25000$)$.

In particular, the analogue readout enables the use of charge-centroid (CC) and $\mu$ TPC algorithms to reconstruct the position, as described in details in Ref. 5 and 7. Two different kinds of prototypes have been developed and tested since 2014: planar triple GEM prototypes of small size and, more recently, a cylindrical prototype of real size, which was intended to serve as one of the CGEM-IT layers. All the prototypes were studied with pions and muons at the $\mathrm{H} 4$ beam line of SPS at CERN, using a dipole magnet with a B field up to $1.5 \mathrm{~T}$. Study of the spatial resolution was performed in various conditions: using two different gas mixtures, several working voltages, magnetic field on and off, and different incident angles of the particles.

In Figure 1 are shown the spatial resolutions of planar prototypes obtained with the $\mathrm{CC}$ and $\mu$ TPC algorithms, as a function of incident angle (B field off) (left) and of the B field (with orthogonal tracks) (right). It turns out that each algorithm succeeds where the other fails in providing good spatial resolution. Nevertheless, using the planar prototypes, it has been proven [8] that a combination of the two algorithms allows keeping the spatial resolution stable at $\sim 130 \mu \mathrm{m}$ in the full range of incident angles with magnetic field on. This spatial resolution is the best achieved worldwide with triple GEM detectors operating in a high magnetic field of $1 \mathrm{~T}$.

CGEM-IT must be built and fully tested to install in the BESIII spectrometer in the summer of 2018 .

\section{TIGER, a new ASIC for GEM readout electronics}

To implement the analogue readout, a custom front-end ASIC with 64-channels has been designed. The Torino Integrated GEM Electronics for Readout (TIGER) ASIC [9], fabricated with UMC 110-nm CMOS technology, provides charge and time measurements with a fully digital 
output. It has been designed in accordance with the detector requirements, to operate with a sensor capacitance of up to $100 \mathrm{pF}$, a linear dynamic range between 1 and $50 \mathrm{fC}$, an event rate of 60 $\mathrm{kHz} / \mathrm{channel}$, a time resolution better than $10 \mathrm{~ns}$, and a power consumption of $\sim 10 \mathrm{~mW} / \mathrm{channel}$. Moreover, the digital back-end of the chip is protected for single-event upset (SEU).
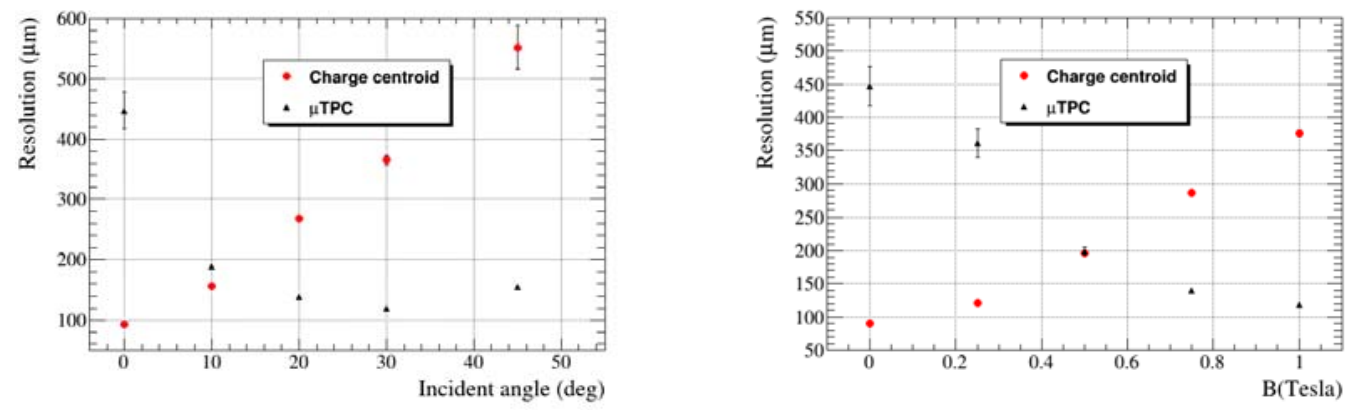

Figure 1. Spatial resolutions with $\mathrm{CC}$ and $\mu \mathrm{TPC}$ algorithms (Left) vs. incident angle from Ref. 5 and (Right) vs. magnetic field from Ref. 4.

A detailed description of TIGER can be found in Ref. 9. Each channel consists of a chargesensitive amplifier followed by two shapers coupled to low-offset discriminators. The peaking time of one shaper is optimized for the time measurement; the peaking time of the other shaper is longer to enable a better integration and optimization of the signal-to-noise ratio for the charge measurement. The very front-end stage is followed by a mixed-mode back-end, with low-power TDCs and Wilkinson ADCs with derandomizing buffers to digitize the time-of-arrival and the charge of the input signal. The back-end design is an updated development of the TOFPET2 chip [10] for medical applications.

The first tapeout in silicon of the TIGER prototype was performed in May 2016, and electrical characterization began in October 2016. Test of the back-end electronics shows good performance. The Sample\&Hold circuit, used for charge measurement, was tested by means of a known input charge and it showed a very good linearity (less than $0.2 \%$ ) for the dynamic range of interest. The quantization error of the TDCs used for the time measurement was less than 50 ps r.m.s., therefore time resolution of the system will be limited only by the sensor and front-end amplifier response.
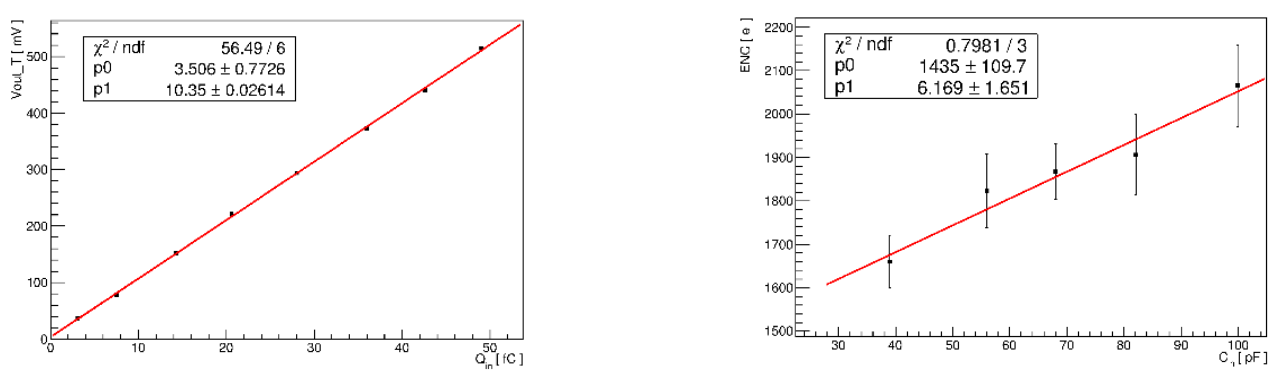

Figure 2. (Left) Gain measurement in one channel from Ref. 9. (Right) ENC of slow shaper output vs capacitance.

The assessment of the analogue front-end was performed for the two branches. The jitter of the time branch was measured as a function of different input capacitances and it was below $4 \mathrm{~ns}$ 
for an input charge of $3 \mathrm{fC}$ with a capacitance of $100 \mathrm{pF}$. A direct measurement of the amplifier gain in one of the channels was performed, as shown in Figure 2(left). An average value of 10.4 $\mathrm{mV} / \mathrm{fC}$ was found, in good agreement with the value of $11 \mathrm{mV} / \mathrm{fC}$ expected from the chip's postlayout simulation. Figure 2(right) shows the equivalent noise charge (ENC) of the slow shaper output of the energy branch, as a function of input capacitance. A value of about 2100 electrons r.m.s. at $100 \mathrm{pF}$ was found, about $50 \%$ greater than that expected from simulations. The root cause of such extra noise was identified in the bias conditions of the holder circuit and an improved design has been done for the final engineering run.

\section{Conclusions}

A cylindrical GEM detector for the BESIII experiment with new features is under construction to replace the inner part of the drift chamber, compromised by aging. The analogue readout of each strip, by means of a custom ASIC in 110-nm CMOS technology, will allow to achieve the required spatial resolution of $130 \mu \mathrm{m}$. Two different readout algorithms were tested with planar triple-GEM prototypes and they look promising. The characterization of the first ASIC prototype shows the overall performance is adequate for the GEM detector and the final engineering run is ongoing with minor revisions.

\section{Acknowledgements}

The research leading to these results was performed within the BESIIICGEM project, funded by the European Commission in the call H2020-MSCA-RISE-2014.

\section{References}

[1] M. Ablikim et al. (BESIII Collaboration), Design and construction of the BESIII experiment, NIM A 614 (2010) 35

[2] F. De Mori (BESIII Collaboration), Light meson spectroscopy at BESIII, in these proceedings

[3] MY. Dong et al., Aging effect in the BESIII drift chamber, Chinese Phys. C 40 (2016) 016001

[4] S. Marcello et al., A new inner tracker based on GEM detectors for the BESIII experiment, in proceedings of CRETE 17 - International Conference on Applications of Nuclear Techniques

[5] R. Farinelli et al., Development and test of a $\mu$ TPC cluster reconstruction for a triple GEM detector in strong magnetic field, in 2016 IEEE NSS/MIC Conference Record, [physics.ins-det/1707.04456]

[6] E. De Lucia, KLOE-2 inner tracker: the first cylindrical GEM detector, in these proceedings

[7] L. Lavezzi et al., The cylindrical GEM inner tracker of the BESIII experiment: prototype test beam results, JINST 12 (2017) C07038 [physics . ins - det/1706 . 0248]

[8] I. Garzia et al., GEM detector performance with innovative micro-TPC readout in high magnetic field, in proceedings of ANIMMA2017

[9] M. Da Rocha Rolo et al., A custom readout electronics for the BESIII CGem detector, JINST 12 (2017) C07017 [physics.ins-det/1706 . 02267]

[10] A. Di Francesco et al., TOFPET2: a high-performance circuit for PET time-of-flight, NIM A 824 (2016) 194 